\title{
Regulation of Opioid Tolerance by let-7 Family MicroRNA Targeting the $\mu$ Opioid Receptor
}

\author{
Ying He, Cheng Yang, Chelsea M. Kirkmire, and Zaijie Jim Wang \\ Department of Biopharmaceutical Sciences, University of Illinois, Chicago, Illinois 60612
}

\begin{abstract}
MicroRNA has emerged as a critical regulator of neuronal functions. This study aimed to test whether let-7 microRNAs can regulate the $\mu$ opioid receptor (MOR) and opioid tolerance. Employing bioinformatics, we identified a let-7 binding site in the 3 ' -untranslated region (UTR) of MOR mRNA, which was experimentally confirmed as a direct target of let-7. The repressive regulation of MOR by let-7 was revealed using a LNA-let-7 inhibitor to knockdown let-7 in SH-SY5Y cells. Conversely, morphine significantly upregulated let-7 expression in SH-SY5Y cells and in a mouse model of opioid tolerance. The LNA-let-7 inhibitor decreased brain let-7 levels and partially attenuated opioid antinociceptive tolerance in mice. Although chronic morphine treatment did not change overall MOR transcript, polysome-associated mRNA declined in a let-7-dependent manner. let-7 was identified as a mediator translocating and sequestering MOR mRNA to P-bodies, leading to translation repression. These results suggest that let-7 plays an integral role in opioid tolerance.
\end{abstract}

\section{Introduction}

Opioid drugs, such as morphine, are a class of powerful analgesics that are used for treating many forms of acute and chronic pain. However, their chronic use has been limited by undesirable effects such as drug tolerance, opioid-induced pain, and opioid dependence. The cellular mechanisms underlying the development of opioid tolerance are not entirely understood. The $\mu$ opioid receptor (MOR) is the primary receptor responsible for opioids' analgesia and antinociceptive tolerance (Matthes et al., 1996). The long $3^{\prime}$-untranslated region (3'-UTR) of MOR mRNA (Ide et al., 2005; Han et al., 2006) is of great interest since this region may contain elements for the posttranscriptional regulation of receptor expression, such as altering the stability of mRNA, influencing translational efficiency and controlling mRNA transport. Indeed, it was reported that human MOR expression was increased after a 712 bp sequence, immediately downstream of the stop codon, was removed (Zöllner et al., 2000).

MicroRNAs (miRNAs) are small noncoding RNA molecules that exert their functions through base-pairing with partially complementary sequences in the $3^{\prime}$-UTR of target mRNAs, resulting in decreased polypeptide formation from those mRNAs. Since the discovery of the first miRNA, lin-4 in Caenorhabditis elegans, hundreds of miRNAs have been identified from humans to viruses, which have provided a crucial and pervasive layer of posttranscriptional gene regulation (Ambros, 2004). The nervous system is a rich source of miRNA expression, with a diversity

Received May 9, 2010; revised June 18, 2010; accepted June 22, 2010.

This work was supported in part by National Institutes of Health (NIH) Grants K07 AT003647 and R01 HL098141. We thank Ellie Jhun for experimental assistance, Dr. Xiaolong He for helpful discussions, and Dr. Williams T. Beck for reviewing the manuscript. C.M.K. was supported by a NIH predoctoral training grant (GM070388). Y.H. was supported by a University of Illinois predoctoral scholarship. Z.J.W. acknowledges scientific discussion with Dr. Lili X. Wang and Dr. Steven T. Rosen of Northwestern University that inspired this work.

Correspondence should be addressed to Dr. Zaijie Jim Wang, Department of Biopharmaceutical Sciences, University of Illinois, 833 S. Wood Street, MC865, Chicago, IL 60612. E-mail: zjwang@uic.edu.

DOI:10.1523/JNEUROSCI.2419-10.2010

Copyright $\odot 2010$ the authors $\quad 0270-6474 / 10 / 3010251-08 \$ 15.00 / 0$ of miRNA functions in fundamental neurobiological processes including neuronal development, plasticity, metabolism and apoptosis (Kosik, 2006). Activity-induced protein synthesis is critical for long-lasting synaptic plasticity and is subject to tight controls. In this study, we tested the hypothesis that let-7 family miRNAs contribute to the development of morphine tolerance.

\section{Materials and Methods}

Constructs, transfection, and luciferase assay. The 3'-UTR of MOR mRNA was amplified from a pcDNA1 clone of the human $\mu$ opiate receptor cDNA (L25119) (Wang et al., 1994) with the following pair of primers: forward primer: 5 '-TAGGCGATCGCTCGAGCTGCTCCGTTGCCCTAA- $3^{\prime}$ and reverse primer 5' - TTGCGGCCAGCGGCCGCACAAACCACTGAAAGCCTAA-3'. The 753 bp fragment was then cloned into the psiCHECK-2 vector (Promega) immediately downstream of the Renilla luciferase reporter gene using the In-Fusion Advantage PCR cloning method (Clontech). The mutated 3'-UTR of MOR mRNA, in which the sequence complementary to let- 7 seed sequence ( $5^{\prime}$-CTACCTC$\left.3^{\prime}\right)$ was truncated, was generated by delete mutation using the QuikChange Lightning Site-Directed mutagenesis method (Stratagene). The sequences of the psiCHECK/MOR1UTR and the psiCHECK/MOR1UTRmut were verified by direct sequencing. For the reporter gene assay, HEK293 cells were seeded in 96-well plates $24 \mathrm{~h}$ before transfection and transiently transfected with the psiCHECK/ MOR1UTR and psiCHECK/MOR1UTRmut constructs with or without either the LNA-let-7 inhibitor or scrambled control using Lipofectamine 2000 (Invitrogen). After $48 \mathrm{~h}$, cells were lysed to determine the Renilla and firefly luciferase activity according to the manufacturer's instructions (Promega). The Renilla luciferase activity was normalized to the activity of the intraplasmid transfection control firefly luciferase. All experiments were performed in triplicates.

Quantitative real-time PCR. Total RNA was extracted using the TRIzol reagent (Invitrogen). The mirVana miRNA Isolation Kit (Ambion) was used to isolate RNA fractions enriched for the small RNA species. The enriched RNA (15 ng) and mirVana quantitative real-time PCR primer sets (Applied Biosystems) specific for each miRNA (let-7a, let-7c, or let-7g) or for the internal control U6 snRNA were used to determine the expression of each miRNA by real-time PCR $(7900 \mathrm{HT}$ Fast Real-Time PCR System, Applied Biosystems). 
Western blot analysis. Western blot analysis was performed as previously described (Tang et al., 2006) using the anti- $\mu$ opioid receptor antibody (1:1000; Neuromics). The expression of $\beta$-actin was similarly determined from the same blots using a monoclonal antibody (1:10,000; Sigma).

Animals and behavioral testing. Male ICR mice (20-25 g, Harlan Industries) were used in this study. Tolerance to opioids was induced by implanting (subcutaneously) morphine pellets [1× $75 \mathrm{mg}$ of morphine base/pellet/mouse; control mice received placebo pellets (National Institute on Drug Abuse, Bethesda, MD)] (Tang et al., 2006). Morphine tolerance was determined by measuring basal nociception and morphine antinociception using cumulative test doses of morphine $(1-10 \mathrm{mg} / \mathrm{kg})$ in a $52^{\circ} \mathrm{C}$ tail-immersion test (Tang et al., 2006). All experimental procedures were performed in accordance with the National Institutes of Health guidelines after approval by the Animal Care Committee of the University of Illinois at Chicago.

In situ hybridization and immunofluoresence. In situ hybridizations for let-7 or MOR mRNA were performed using fluorescein- or Texas red-labeled LNA probes complementary to all members of let-7 or MOR coding region, respectively. let-7 probe: $5^{\prime}$-AACTATGCAACCTACTACCTCA-3'; MOR probe: 5'-ATGAACATTACGGGCAGACCA-3'. Hybridizations were performed as described previously (Pillai et al., 2005; Obernosterer et al., 2007). For immunofluorescence analysis, the antibody for hDcpla (Santa Cruz Biotechnology) and MOR were used at 1:500 and 1:5000 dilutions, respectively. Secondary anti-goat and antimouse antibodies labeled with Alexa 488 and Alexa 594 fluorochromes (Invitrogen), respectively, were used at 1:500 dilutions.

Polysome analysis. The polysomal RNA was isolated as described previously (Mukhopadhyay et al., 2003). The primer sequences for reverse transcriptase PCR (RT-PCR) were as follows, MOR forward: 5'-CTCTTCAGCCATTGGTCTT-3'; MOR reverse: $5^{\prime}$-CCATCCTGGTGATCCTTC-3' ${ }^{\prime} \beta$-actin forward: $5^{\prime}$-CACCAACTGGGACGACAT- ${ }^{\prime}$; $\beta$-actin reverse: $5^{\prime}$-CATACTCCTGCTTGCTGATC-3'.

Data analysis and statistics. All data are expressed as mean \pm SEM. The statistical comparisons were analyzed by ANOVA followed by Dunnett's $t$ test (multiple groups) or Student's $t$ test (two groups).

\section{Results}

Predicted interaction of let-7 miRNAs with $3^{\prime}$-UTR of MOR mRNA

We first performed bioinformatic analyses to identify potential miRNAs that may interact with MOR. Three different algorithms and databases were used to predict putative miRNAs that can target the 3 '-UTR regions of the human (Ide et al., 2005) and mouse (Han et al., 2006) MOR genes: PicTar (http://pictar.mdc-berlin.de/); TargetScan 5.0 (http:// www.targetscan. org/); and miRanda (http://www.microrna.org/ microrna/getGeneForm.do). The predicted miRNAs were ranked according to the number of putative target sites and sum

A

B

C

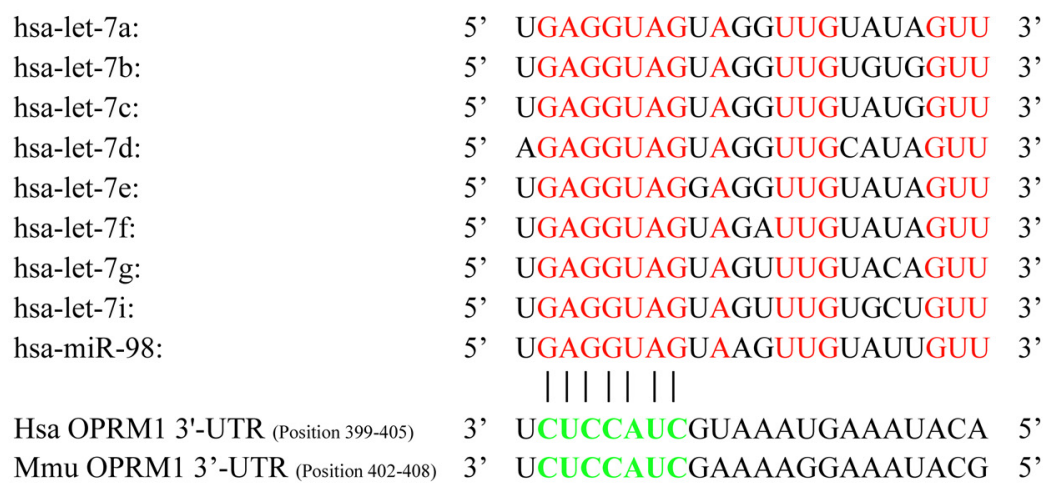
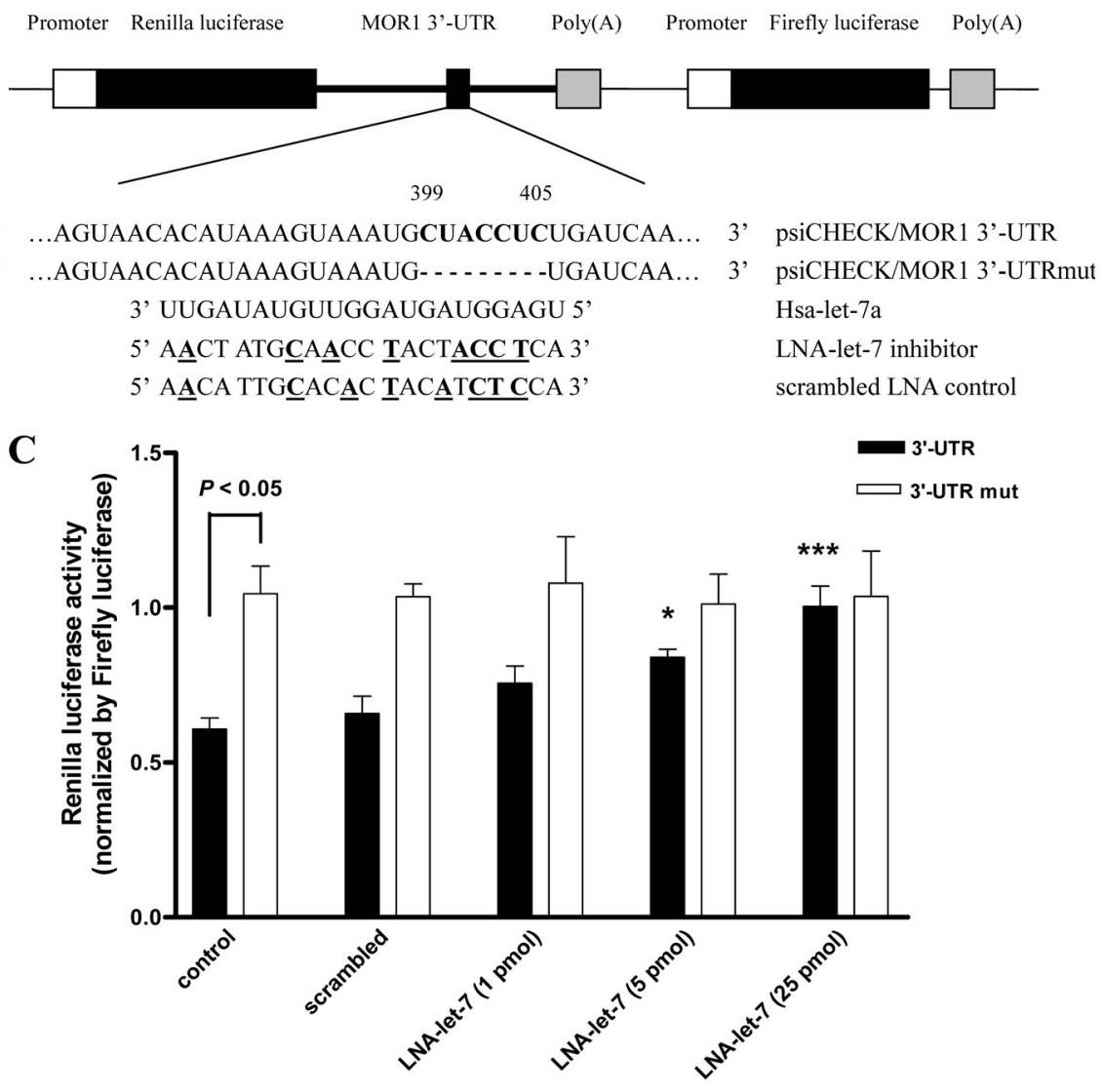

Figure 1. Interaction of let-7 with the $3^{\prime}-U T R$ of the MOR. A, Sequence alignment of the let-7 family miRNAs and the predicted sequence pairing with a region of MOR mRNA $3^{\prime}$-UTR. The nucleotides in red are the positions that are identical among all members of the let-7 family. The predicted MOR mRNA $3^{\prime}$-UTR sequences that may interact with let-7 seed match are in green (bold). $B, A$ schematic plot of the luciferase constructs. Solid bar indicates the location of the putative let-7 binding site within the $3^{\prime}$-UTR of MOR mRNA. The LNA-let-7 inhibitor and scrambled-LNA control are shown below the let-7a alignment with the position of LNA nucleotides underlined in bold. C, Luciferase reporter assays were performed in HEK293 cells transfected with the reporter plasmids psiCHECK/MOR1UTR or psiCHECK/MOR1UTRmut together with the LNA-let-7 inhibitor $(0,1,5,25 \mathrm{pmol})$ or scrambled-LNA control ( $25 \mathrm{pmol}$ ). The Renilla luciferase activity was normalized to the internal control firefly luciferase activity. ${ }^{*} p<0.05,{ }^{* * *} p<0.001$ vs the $3^{\prime}$-UTR control $(n=3)$.

of alignment scores determined by both seed match type and seed match context. (See supplemental Table S1, available at www. jneurosci.org as supplemental material, for a list of miRNAs with top scores.) Comparing the putative miRNAs generated from these programs, we found that the let-7 family of miRNAs consistently scored the highest on every list. In the human and mouse, the let-7 miRNA family consists of nine distinct, mature 22-nucleotide sequences that differ only at one to four positions 
from the canonical let-7a. An exact match to the seed sequence of let-7 (positions 2-8, Fig. 1A) was found in MOR 3'-UTR 399405bp (human) or 402-408 bp (mouse) downstream from the stop codon of MOR1, the most abundant transcript for MOR (Ide et al., 2005; Han et al., 2006).

\section{Functional targeting of the predicated 3 '-UTR of MOR mRNA by let-7}

To validate the in silico prediction that members of the let-7 miRNA family can interact with the $3^{\prime}$-UTR of MOR mRNA at the predicted positions, a 753 bp human MOR 3'-UTR DNA fragment containing the putative let-7 interaction sequence was constructed and subcloned into the psiCHECK-2 vector (psiCHECK/MOR1UTR). A deletion mutant of this $3^{\prime}$-UTR fragment lacking the putative let-7 sequences (Fig. $1 B$ ) was also constructed (psiCHECK/MOR1UTRmut). These constructs were transiently transfected into the HEK293 cells that endogenously express let-7 miRNAs. Transfection efficacy was normalized by the internal control firefly luciferase activity on psiCHECK-2, which is not affected by the subcloned sequences. The Renilla luciferase activity in the psiCHECK/MOR1UTRtransfected HEK293 cells was only $60 \%$ of those transfected with psiCHECK/MOR1UTRmut ( $p<0.05$, Fig. $1 C)$. These data suggested that psiCHECK/MOR1UTR contained a repressor sequence that was not present in psiCHECK/MOR1UTRmut.

If indeed the difference was due to the interaction with let-7 miRNAs, it is expected that inhibiting endogenous let-7 activity in HEK293 cells would restore the Renilla luciferase activity. To inhibit let-7, we applied antisense oligodeoxynucleotides to knock down the expression (Fig. 1B). This LNA-let-7 inhibitor contains eight locked nucleic acid (LNA) nucleotides at positions shared by all nine members of the let-7 family (Boyerinas et al., 2008). For the negative control, scrambled oligodeoxynucleotides were designed to have the same nucleotide content including LNA nucleotides (Fig. 1B) (Wahlestedt et al., 2000). The LNAlet-7 inhibitor $(1,5$, or $25 \mathrm{pmol})$ or scrambled control $(25 \mathrm{pmol})$ was cotransfected into the HEK239 cells together with the psiCHECK/MOR1UTR or the psiCHECK/MOR1UTRmut. The LNA-let-7 inhibitor increased the reporter gene activity in a dose-dependent manner. Cotransfection with 25 pmol of LNA-let-7 inhibitor completely restored the luciferase gene repression by psiCHECK/MOR1UTR. In contrast, the LNAlet-7 inhibitor did not change the luciferase signal when cotransfected with the psiCHECK/MOR1UTRmut that lacks the let-7 binding sequence (Fig. $1 C$ ). The scrambled control did not alter the reporter gene activity. These data are in agreement with the hypothesis that let-7 can bind to the putative sequence on the 3'-UTR of MOR mRNA.

Identifying MOR as a direct target of let-7 in SH-SY5Y cells To determine the functional relevance of the interaction between let-7 and the MOR mRNA 3'-UTR sequence, we tested whether the inhibition of let-7 by the LNA-let-7 inhibitor could increase MOR expression in human neuroblastoma SH-SY5Y cells that endogenously express the receptor (Yu and Sadée, 1988). MOR expression was determined by Western blotting. When SH-SY5Y cells were transfected with increasing amounts of the LNA-let-7 inhibitor, MOR expression was upregulated in an antisense dosedependent fashion (Fig. 2). Transfection of the scrambled control $(250 \mathrm{pmol})$ did not alter receptor expression. Since knocking down let-7 increased the MOR expression, these data suggest that
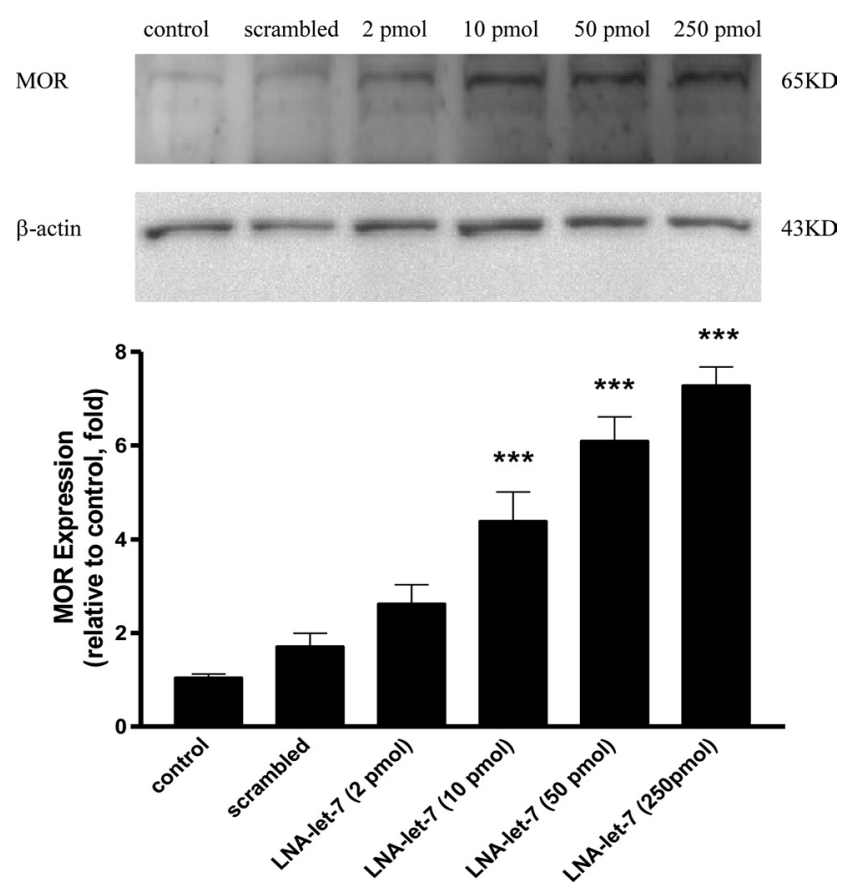

Figure 2. Constitutive repression of MOR expression by let-7 miRNAs in SH-SY5Y cells. SHSY5Y cells were transfected with the LNA-let-7 inhibitor $(0,2,10,50,250$ pmol) or scrambledLNA control (250 pmol). Cells were lysed for Western blot analysis $48 \mathrm{~h}$ later. The anti-MOR antibody did not detect $65 \mathrm{kDa}$ MOR bands in the null deletion mice (supplemental Fig. S1, available at www.jneurosci.org as supplemental material). Histogram graph was constructed from the representative figure shown and two other experiments. ${ }^{* * *} p<0.001$ vs the control.

not only is MOR a target of let-7 but the endogenous expression of MOR is under constitutive suppression by let-7.

Upregulation of let-7 and downregulation of MOR by chronic treatment with morphine in SH-SY5Y cells

Since prolonged exposure to morphine is known to induce cellular tolerance to opioids in SH-SY5Y cells that endogenously express MOR and let-7 (Yu and Sadée, 1988), we next determined whether let-7 expression can be regulated by morphine in these cells. The genomic organization of the let-7 family is highly conserved and clustered into several patterns (Roush and Slack, 2008); we chose let-7a, let-7c, and let-7g as representative members of the family. Cells were treated with morphine ( $1 \mu \mathrm{M}$ for 24 , or $48 \mathrm{~h}$ ) to induce cellular tolerance before they were harvested for quantitative real-time PCR analysis of the expression of let-7a, c, and g. As shown in Figure 3, the expression of all three let-7 miRNAs was significantly increased by chronic treatment with morphine in SH-SY5Y cells $(p<0.05)$. It is also known that retinoic acid promotes neuronal differentiation and increases MOR expression, as well as the magnitude of cellular tolerance to morphine in SH-SY5Y cells (Yu and Sadée, 1988). After pretreatment with retinoic acid ( $5 \mu \mathrm{M}$, for $6 \mathrm{~d})$, chronic morphine induced a similar, but greater, upregulation of let-7a, $c$, and $g$ expression in SH-SY5Y cells. This suggests that retinoic acid potentiated the effect of morphine in the upregulation of let-7. In contrast, the brain-specific miR-134 was not regulated by morphine (Fig. 3D). These data strongly indicate that let-7 is regulated by morphine during the development of cellular opioid tolerance in SH-SY5Y cells.

In these studies, MOR expression was also determined in $\mathrm{SH}$ SY5Y cells after treatment with morphine for 24 or $48 \mathrm{~h}$. The expression of MOR was significantly reduced after $24 \mathrm{~h}$ treatment 
A

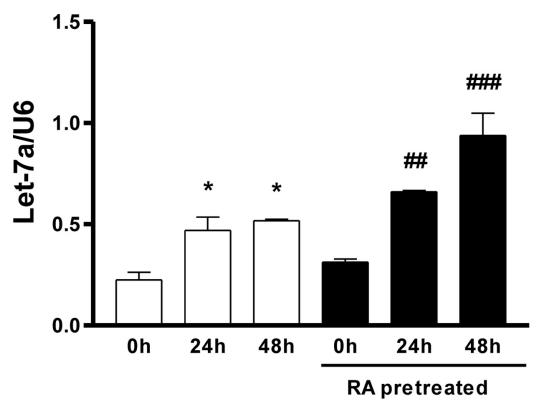

D

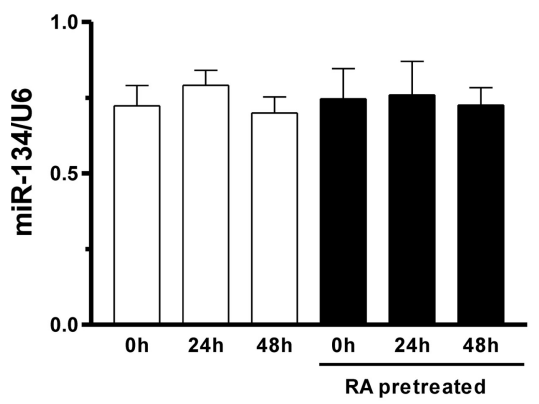

B

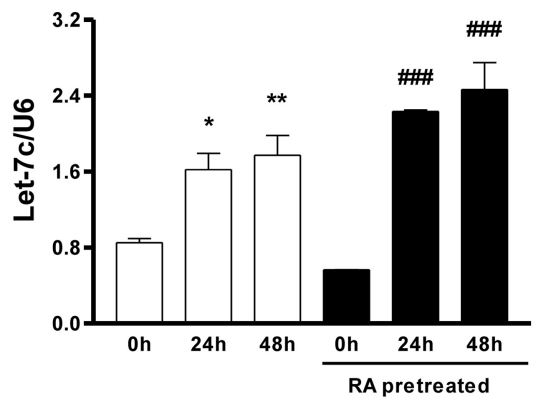

$\mathbf{E}$

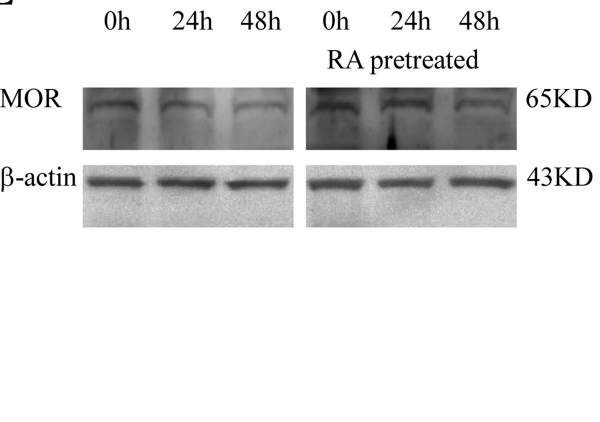

C

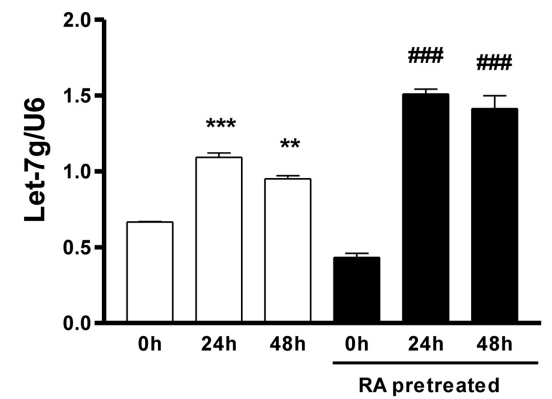

$\mathbf{F}$

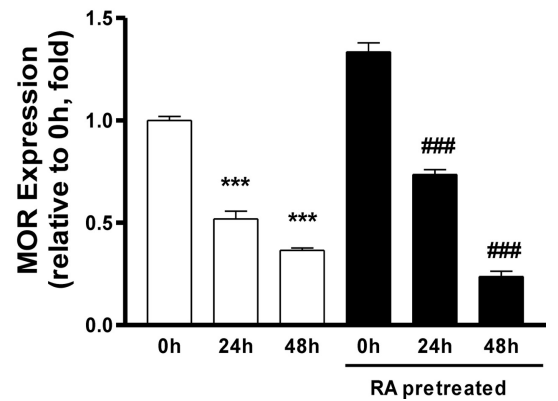

Figure 3. Increased let-7 expression and decreased MOR expression in SH-SY5Y cells during the development of opioid tolerance. SH-SY5Y cells with or without retinoic acid (RA) pretreatment $(5 \mu \mathrm{m}, 6 \mathrm{~d})$ were incubated with morphine $(1 \mu \mathrm{m}, 24-48 \mathrm{~h})$ to induce a tolerant state. The transcripts of let-7a $(\boldsymbol{A})$, let-7c $(\boldsymbol{B})$, and let-7g (C), but not miR-134 (D), were increased as determined by the real time RT-PCR. MOR expression was decreased as measured by Western blot analysis $(\boldsymbol{E}, \boldsymbol{F}) .{ }^{*} p<0.05,{ }^{* *} p<0.01,{ }^{* * *} p<0.001$ compared with 0 h without RA; ${ }^{\# \#} p<0.01,{ }^{\# \# \#} p<0.001$ compared with 0 h with $\mathrm{RA}(n=3)$.

with morphine and even more after $48 \mathrm{~h}$ as determined by Western blot analysis (Fig. 3 E, F). Therefore, let-7 and MOR expression is inversely regulated by morphine during the development of opioid tolerance in SH-SY5Y cells.

\section{Upregulation of let-7 by chronic} treatment with morphine in mice To determine the in vivo relevance of let-7 regulation by morphine during the development of opioid tolerance, we examined the expression of let-7 miRNAs in a mouse model of opioid tolerance (Tang et al., 2006). Brain expression of let-7 increased gradually over time after morphine treatment (1× $75 \mathrm{mg}$ of morphine/pellet/ mouse, s.c.) (Fig. $4 A-C$ ), correlating with the development of antinociceptive tolerance to morphine (Fig. $4 E$ ). Significant upregulation was observed on day $3(p<$ $0.05)$. On day 5 , when tolerance was fully developed, the expression of let-7a, $c$, and g increased by $4.0-$, 4.5-, and 4.7-fold, respectively ( $p<0.001$ compared with day 0 ). Moreover, upregulation of let-7 occurred in MOR-expressing cells (78/85) in the mouse, but not in MOR-negative cells (0/65), as determined by in situ hybridization (Fig. 5). As in SH-SY5Y cells, miR-134 was not regulated by morphine in mice (Fig. 4D). These data collectively demonstrated a temporal correlation between let-7 upregulation and the development of opioid tolerance. It is plausible that chronic morphine exposure may increase the expression of let-7 which, in turn, downregulates MOR ex-
A

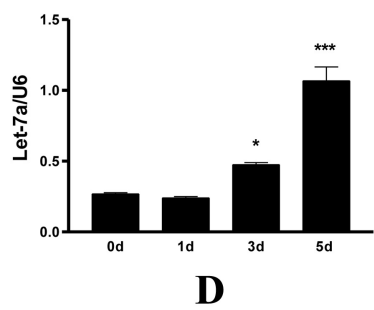

B

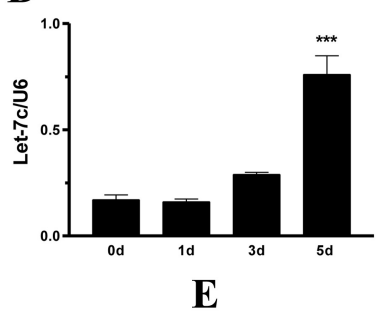

C

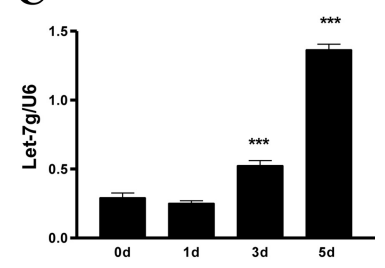

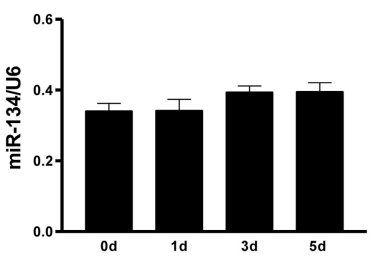

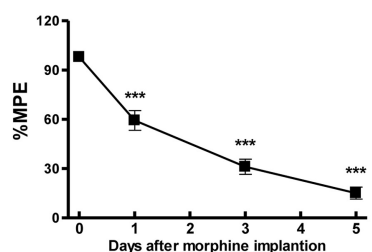

Figure 4. Increased let-7 expression in the mouse brain during the development of opioid tolerance. Mice were implanted with morphine pellets to induce opioid tolerance. On day $0,1,3$ and 5 , brain let-7a $(\boldsymbol{A})$, let-7c(B), let-7g ( $)$, and miR-134 (D) transcripts were analyzed by quantitative RT-PCR. The development of opioid tolerance was confirmed by following significantly reduced morphine-antinociception in a tail-flick assay $(\boldsymbol{E}) .{ }^{*} p<0.05,{ }^{* * *} p<0.001$ vs day 0 group $(n=5)$.

pression, contributing to the behavioral manifestation of opioid tolerance.

\section{Reversal of opioid tolerance by knocking down of let-7} expression in mice

The data so far have convincingly demonstrated a consistent correlation of let-7 upregulation with the development of opioid tolerance in both cellular and rodent models. To further examine a causative role of let-7 in leading to opioid tolerance beyond the temporal and dose correlations, we directly targeted let-7 in the mouse model of opioid tolerance. First, the LNA-let-7 inhibitor 


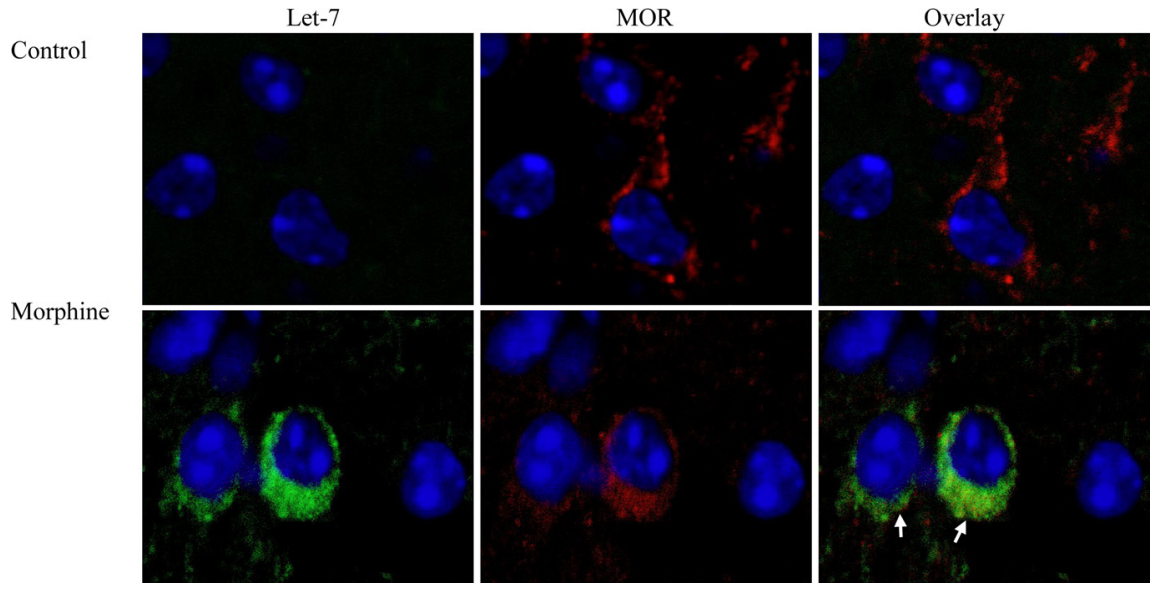

Figure 5. Fluorescence in situ hybridization (FISH) of let-7 before and after the treatment with morphine $(75 \mathrm{mg} / \mathrm{pellet} /$ mouse). The brain cortex was used for the analysis of let-7 mRNA by FISH. Samples were costained using anti-MOR antibody (1:5000) to determine the level of let-7 in MOR-expressing cells. The let-7 transcript was increased after morphine treatment. Moreover, the increase was observed in MOR-expressing cells (arrows indicate representative cells with costaining of MOR and let-7), but not in MOR-negative cells (only DAPI nuclear staining, but no MOR staining). Fifteen prefrontal cortex slices from 3 mice were studied for each condition. In the control group, 78 MOR-expressing cells and 60 MOR-negative cells were imaged. In the morphine group, let-7 upregulation occurred in 78/85 MOR-expressing cells; no cell showed let-7 response in MOR-negative cells (0/65).
A

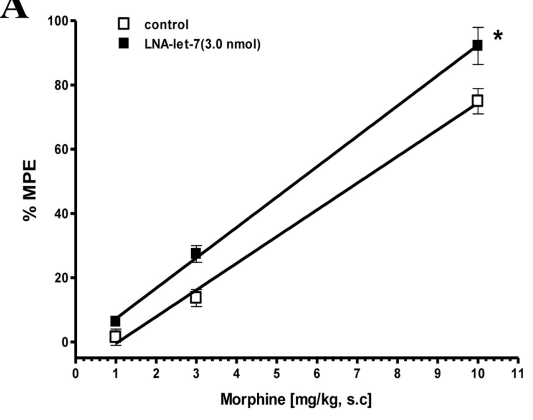

C

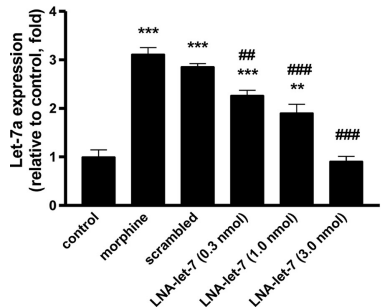

D

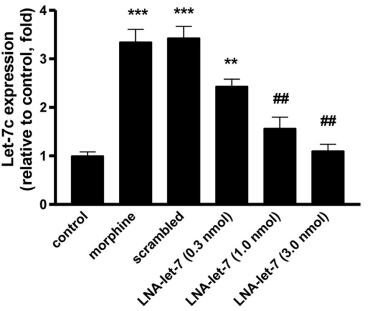

$\mathbf{E}$

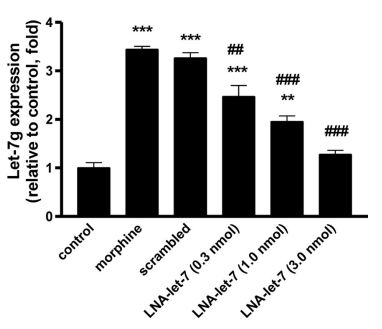

Figure 6. Effect of the LNA-let-7 inhibitor on morphine antinociception, morphine antinociceptive tolerance, and let-7 expression in mice. $\boldsymbol{A}$, The LNA-let-7 inhibitor potentiated morphine antinociception in naive mice, suggesting constitutive repression of MOR by let-7. $\boldsymbol{B}$, The LNA-let-7 inhibitor dose-dependently attenuated morphine antinociceptive tolerance. $\boldsymbol{C}-\boldsymbol{E}$, Morphineinduced let-7 upregulation was dose-dependently reduced by the LNA-let-7 inhibitor (0.3-3.0 nmol, twice/day, for $3 \mathrm{~d})$. ${ }^{*} p<$ $0.05,{ }^{* *} p<0.01,{ }^{* * *} p<0.001$ v s the control group; ${ }^{*} p<0.05,{ }^{\# \#} p<0.01$, ${ }^{\# \# \#} p<0.001$ vs the morphine group $(n=4)$. doses of test morphine (Tang et al., 2006). There was a significant downward shift of dose-response curves in mice implanted with morphine compared with placebopellet-treated mice (Fig. $6 \mathrm{~B}$ ). In placebopelleted mice, $\mathrm{ED}_{50}$ was $4.3 \pm 0.5 \mathrm{mg} / \mathrm{kg}$ and the maximum antinociceptive effect of morphine reached 94\%. After a $6 \mathrm{~d}$ treatment with morphine, morphine was no longer active in the antinociceptive test and produced 3\% maximum possible effect (MPE), indicative of the development of antinociceptive tolerance to morphine. Paralleling the development of tolerance, the levels of let-7a, $\mathrm{c}$, and g were significantly increased by 3.1-, 3.3-, and 3.4fold, respectively ( $p<0.001$, Fig. $6 C-E)$.

In morphine-pelleted mice that also received the LNA-let-7 inhibitor, however, the expression of let-7 declined in a dose-dependent manner, confirming the expected knocking down of let-7 by the LNA-let-7 inhibitor in vivo. In these mice, morphine antinociception was partially restored, producing $60 \% \mathrm{MPE}\left(\mathrm{ED}_{50}=8.9 \pm\right.$ $1.1 \mathrm{mg} / \mathrm{kg}), 31 \% \mathrm{MPE}\left(\mathrm{ED}_{50}=19 \pm 1.2\right.$ $\mathrm{mg} / \mathrm{kg})$, and $23 \% \mathrm{MPE}\left(\mathrm{ED}_{50}=32 \pm 1.3\right.$ $\mathrm{mg} / \mathrm{kg}$ ) after treatment with $3.0,1.0$, and 0.3 nmol, respectively, of LNA-let-7 inhibitor. Therefore, knocking down let-7 expression partially blocked the development of opioid tolerance. These data together with the inverse correlation between let-7 and MOR expression suggest that let-7 plays an integral role in opioid tolerance.

Translation repression of MOR by let-7 MicroRNAs, when bound to $3^{\prime}$-UTR sequences, are known to affect target mRNA stability and/or translation efficiency (Moss et al., 1997; Wu et al., 2006). We first determined MOR mRNA levels in SH-SY5Y cells following morphine treatment $(1 \mu \mathrm{M}$ for $48 \mathrm{~h})$ and found that total MOR mRNA was not changed (Fig. 7A). This result was in agreement with previous reports from different cell lines or animal models (Brodsky et al., 1995; Johnson et al., 2006). It is, therefore, unlikely that let-7 affected MOR mRNA stability. To investigate whether translation efficiency was inhibited by let-7, we analyzed polysome profiles of MOR mRNAs. was tested in naive mice. Treatment with the LNA-let-7 inhibitor (intracerebroventricularly) for $3 \mathrm{~d}$ slightly, but significantly, potentiated morphine antinociception ( $24 \%$ increase of maximum effect, $p<0.05$ ) (Fig. 6A). These data indicate that there is a certain degree of constitutive repression of MOR by let-7 in vivo, which was also present in SH-SY5Y cells (Fig. 2).

Two days after the subcutaneous implantation of morphine pellets, mice received the LNA-let-7 inhibitor or scrambled control (intracerebroventricularly, twice a day for 3 consecutive days). On day 6, opioid tolerance was examined using cumulative
In contrast to the level of total mRNA, polysome-bound MOR transcript was significantly decreased after morphine treatment $(1 \mu \mathrm{M}$ for $48 \mathrm{~h}$ ) in SH-SY5Y cells (Fig. 7B). As an indicator of translation efficiency, reduced polysomal mRNA would suggest that MOR is repressed at the translation initiation. Furthermore, reduced polysome-bound MOR mRNA was significantly recovered by the LNA-let-7 inhibitor, indicating that MOR translation inhibition seen in opioid tolerance was mediated, at least in large part, by let-7. It has been demonstrated that target mRNA, in complex with miRNAs in the RISC (RNA-induced silencing 


\section{A}

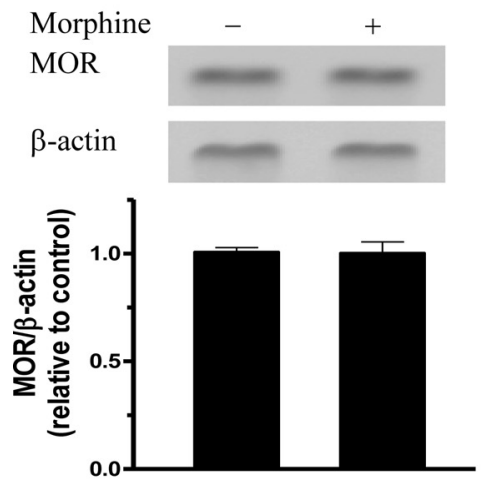

\section{B}

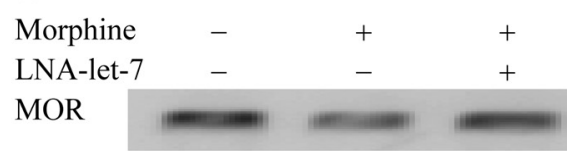

$\beta$-actin
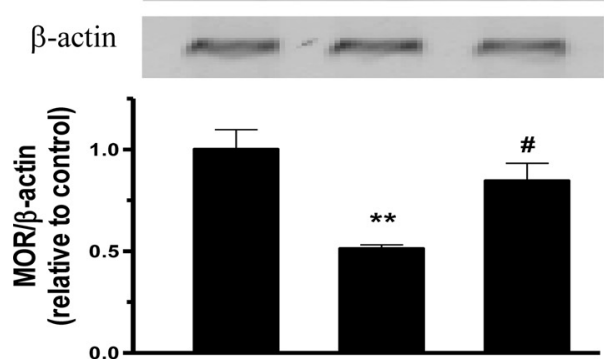

C
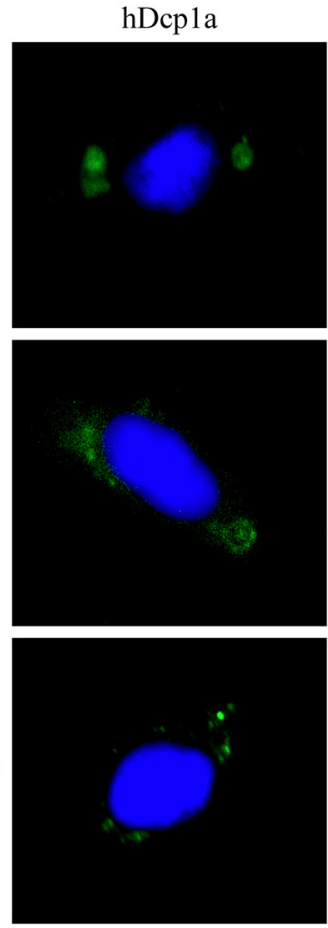

LNA-let-7

Morphine
MOR mRNA
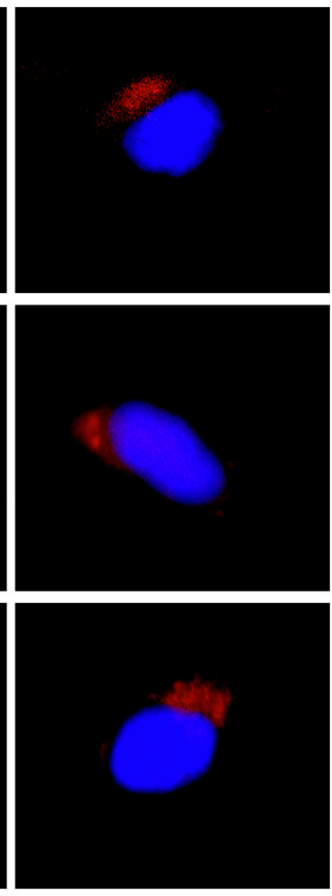
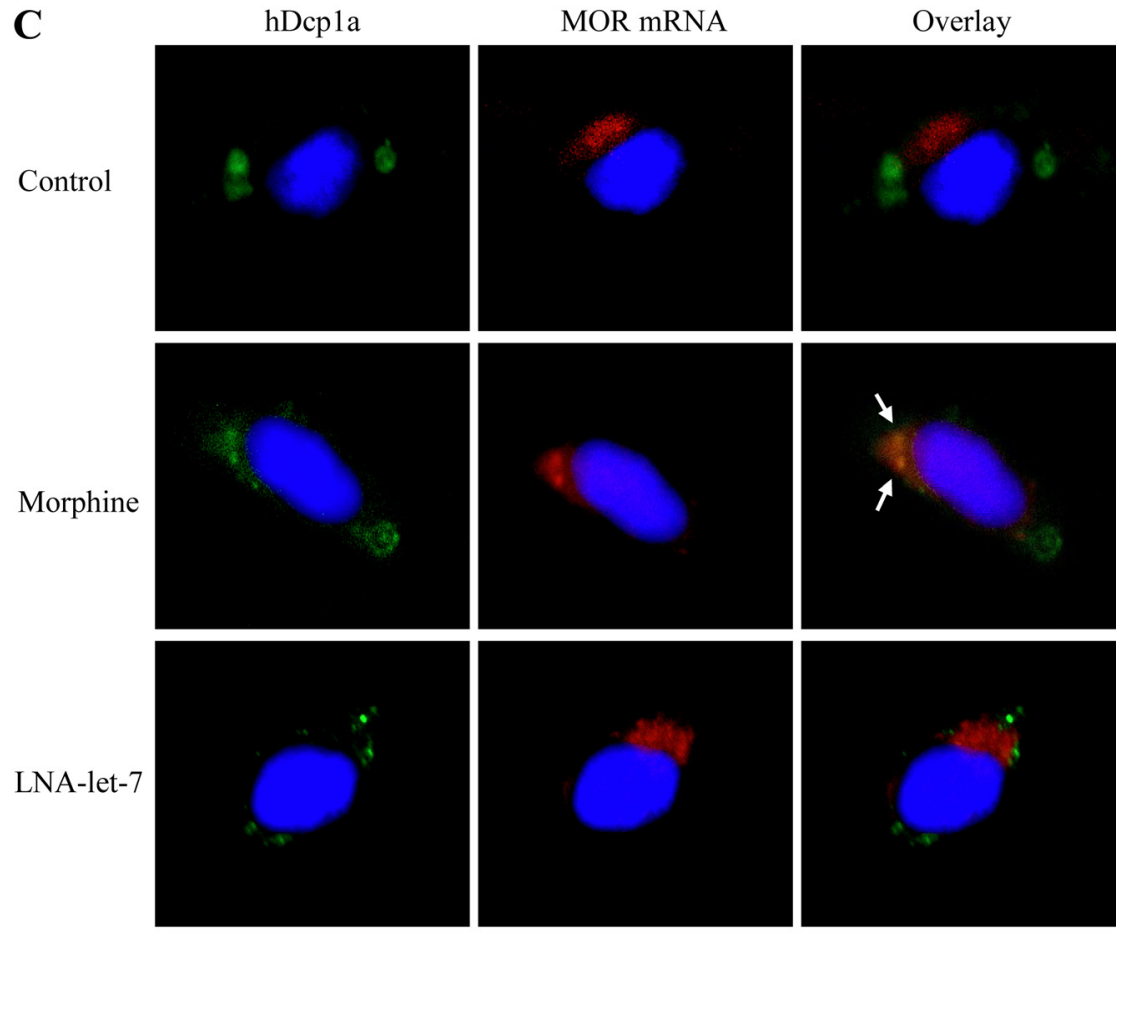

Figure 7. The total and polysome-associated $\mu$ opioid receptor transcript after the treatment with morphine in SH-SY5Y cells. SH-SY5Y cells were treated with morphine (1 $\mu \mathrm{M}, 48 \mathrm{~h}) . \boldsymbol{A}, \mathrm{MOR}$ transcript was not altered by morphine ( $n=5$ each group). $\boldsymbol{B}$, Polysome-associated MOR mRNA was decreased by morphine. The change was significantly attenuated by the LNA-let-7 inhibitor. ${ }^{* *} p<0.01$ vs the control group; ${ }^{\#} p<0.05$, vs the morphine group ( $n=5$ each group). C, Colocalization of MOR mRNA with hDcp1a, a P-body marker, after, but not before, morphine treatment. Blue (DAPI) identifies nuclei.

complex), can be accumulated in the cytoplasmic foci, referred to as processing bodies (P-bodies), thereby reducing polysome mRNA (Pillai et al., 2005; Bhattacharyya et al., 2006). To test this hypothesis, we colabeled MOR mRNA and hDcpla, a P-body marker. Indeed, MOR transcript was colocalized with hDcpla after, but not before, the treatment with morphine (Fig. 7C). Colocalization was abolished by transfecting cells with the LNAlet-7 inhibitor, indicating that P-body enrichment of MOR mRNA was let-7 dependent. These data suggest that let-7 regulates MOR expression by suppressing translation initiation, not by reducing mRNA.

\section{Discussion}

The mechanisms underlying the development of opioid tolerance are not entirely understood. We proposed here that posttranscriptional regulation of MOR by miRNAs may be a molecular mechanism contributing to tolerance. Specifically, this study examined the role of let-7 miRNAs in cellular and animal models of opioid tolerance. Inhibiting let-7 expression was found to increase MOR protein expression in SH-SY5Y cells and the level of let-7 appeared to be inversely correlated with that of the receptor. On the other hand, let-7 expression was regulated by chronic treatment with morphine in SH-SY5Y cells and in mice that were tolerant to opioids. Moreover, knocking down let-7 partially attenuated the antinociceptive tolerance to morphine. These data strongly implicate a repressive role of let-7 on MOR expression, thus contributing to the development of opioid tolerance. let-7 was the first identified human miRNA. Its family members are highly conserved across species in sequence and function. Known biological functions for let-7 include the regulation of stem-cell differentiation, neuromuscular development, and cell proliferation and differentiation (Reinhart et al., 2000; Mansfield et al., 2004; Roush and Slack, 2008). The findings of our study revealed a new role of let-7 in modulating opioid tolerance.

Extensive efforts have been made to elucidate the molecular mechanisms leading to opioid tolerance and drug addiction. Compared with the abundant studies on the promoter and other $5^{\prime}$-UTR regions of MOR, the research on $3^{\prime}$-UTR of its mRNA is limited. However, the especially long $3^{\prime}$-UTR of MOR mRNA suggests that this region may contain physiologically relevant elements for regulating receptor expression by mechanisms such as miRNA targeting. In the present study, comparative bioinformatics predicted MOR to be a potential target of let-7. We observed a robust change of let-7 expression in opioid tolerance and the upregulation of let-7 appeared to be linked with the development of opioid tolerance. On the other hand, we noticed that the LNA-let-7 inhibitor did not fully restore the antinociceptive effect of morphine, even at the highest dose used. At this dose, the LNA-let-7 inhibitor significantly suppressed let-7 expression to premorphine control levels. In addition, there was a discrepancy between let-7 upregulation time course and onset of antinociceptive tolerance. Whereas morphine tolerance was detectable with $24 \mathrm{~h}$ after morphine implantation, let-7 mRNA levels were significantly upregulated $72 \mathrm{~h}$, but not $24 \mathrm{~h}$, after morphine implanta- 


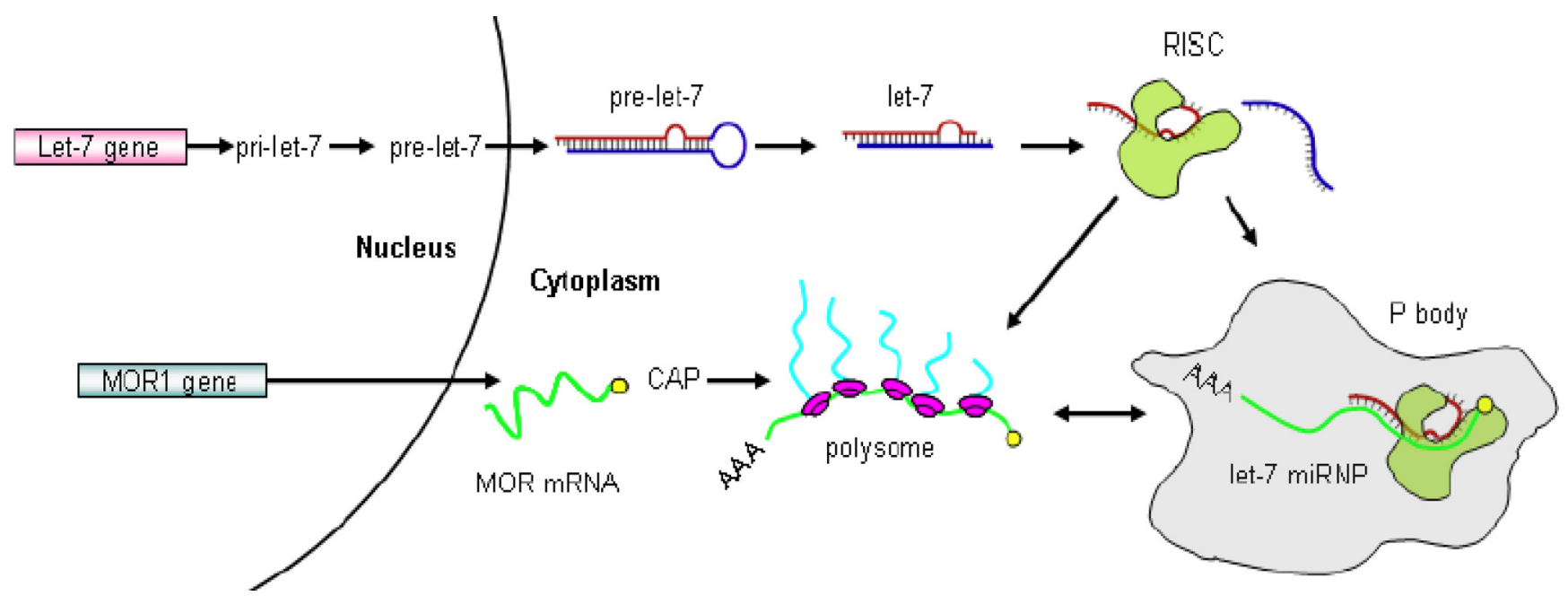

Figure 8. A schematic plot for the proposed mechanism of MOR regulation by let-7. Pri- and pre-let-7 were produced and exported into the cytosol where the mature let-7 was incorporated into the RISC. The latter recruits and sequesters MOR mRNA to P-bodies that are deprived of translational machinery, effectively reducing polysome-bound MOR mRNA and leading to translation repression.

tion. These data suggest that the signaling cascade of opioid tolerance will involve other components in addition to let-7. For example, let-7 will not participate in regulatory mechanisms at the gene replication and transcription levels since miRNAs exhibit their actions at the posttranscriptional level. It is even reasonable to speculate that there are other potential miRNAs acting on the $3^{\prime}$-UTR of MOR mRNA. Recently, another research group found the involvement of miR-23b in the regulation of mouse MOR expression (Wu et al., 2008; Wu et al., 2009). In the current study, we identified let-7 miRNAs as a regulator of human as well as mouse MOR. Moreover, we demonstrated in vivo relevance of let-7 miRNAs in the development of opioid tolerance. It is not known whether miR-23b and let-7 crosstalk during the development of opioid tolerance. The LNA-let-7 inhibitor did not inhibit miR-23b transcript (supplemental Fig. S2, available at www. jneurosci.org as supplemental material). In addition to MOR, it is also possible that let-7 may target other proteins in opioid tolerance. A number of receptors, ion channels, and protein kinases have been implicated in opioid tolerance, such as the NMDA receptors, PKC, and CaMKII (Kieffer and Evans, 2002; Wang and Wang, 2006) which may become potential targets for let-7.

Receptor downregulation as one of mechanisms contributing to opioid tolerance has been previously proposed (Davis et al., 1979; Tao et al., 1987; Bhargava and Gulati, 1990; Bernstein and Welch, 1998; Díaz et al., 2000). Chronic treatment with morphine, similar to the method used in this study, produced a marked decrease in brain MOR density (Davis et al., 1979; Tempel et al., 1988). Downregulation of the high-affinity MOR site in rats has also been reported following continuous infusion of morphine (intrathecally) (Wong et al., 1996) or etorphine (subcutaneously) (Tao et al., 1987). Morphine-induced MOR downregulation was also observed in SH-SY5Y cells, with or without differentiation (Zadina et al., 1993). We found, in this study, that let-7 expression was increased by morphine, with or without retinoic acid pretreatment, in SH-SY5Y cells. Interestingly, the expression of let-7c and $g$ was reduced by retinoic acid in morphine-untreated cells (Fig. 3, $p<0.01$; no significant change on let-7a). These data would suggest that retinoic acid may increase MOR expression, at least in part, through the inhibition of certain let-7 expression. In addition to the receptor downregulation, chronic treatment with morphine has been shown to significantly reduce MOR signaling in sensory neurons and certain brainstem nuclei (Sim et al., 1996; Johnson et al., 2006).

However, MOR downregulation by opioids is not without controversy. First, MOR mRNA has been reproducibly found not to be altered by many different opioid agonists, which appeared to be inconsistent with significant changes in receptor expression. Our current finding, however, has provided an explanation that let-7 can reduce MOR translation without affecting mRNA. Then, there are reports that MOR expression was not altered (Klee and Streaty, 1974; Höllt et al., 1975; Dum et al., 1979) or even upregulated (Holaday et al., 1982; Lewis et al., 1984; Rothman et al., $1986)$ in the brain by various opioids. Some of the discrepancies may be caused by different opioids, doses, treatment methods, different anatomical regions and times sampled, as well as detection methods used in different studies. It has been suggested that MOR downregulation is agonist selective and depends on the agonist's intrinsic efficacy (Koch and Höllt, 2008). The purity and selectivity of radiolabeled ligands used in studies are other potential culprits for conflicting findings. Third, the expectation of a single mechanism causing opioid tolerance may not be realistic. Instead, multiple mechanisms are likely involved in opioid tolerance. MOR downregulation represents one of many mechanisms contributing to opioid tolerance. Indeed, knocking down let-7 only partially restored morphine antinociception.

The specific mechanism of MOR regulation by let-7 was found to be at translation initiation. Based on the experimental data, we would like to propose the following pathway by which let-7 reduces MOR expression (Fig. 8). Upon exporting into the cytosol, mature let-7 is incorporated into RISC that mediates translational inhibition. Recruitment of MOR mRNA to P-bodies by let-7 effectively reduces the polysome-bound transcript. Since P-bodies, where the mRNAs are sequestered or degraded by the decapping enzymes and exonucleases, do not contain the translational machinery, the net outcome of reduced polysome mRNA and recruitment of mRNA to P-bodies is translation repression. A similar mechanism on translation repression has been proposed previously for let-7 in HEK293 and HeLa cells (Pillai et al., 2005).

To our knowledge, this is the first report on the role of a specific miRNA in opioid tolerance in vivo. The repressive regu- 
lation of MOR by let-7 represents one element leading to the development of opioid tolerance. These findings reveal miRNAmediated regulation of MOR which provides a novel direction toward unraveling the complex mechanisms involved in the development of opioid tolerance.

\section{References}

Ambros V (2004) The functions of animal microRNAs. Nature 431: $350-355$.

Bernstein MA, Welch SP (1998) mu-Opioid receptor down-regulation and cAMP-dependent protein kinase phosphorylation in a mouse model of chronic morphine tolerance. Brain Res Mol Brain Res 55:237-242.

Bhargava HN, Gulati A (1990) Down-regulation of brain and spinal cord mu-opiate receptors in morphine tolerant-dependent rats. Eur J Pharmacol 190:305-311.

Bhattacharyya SN, Habermacher R, Martine U, Closs EI, Filipowicz W (2006) Relief of microRNA-mediated translational repression in human cells subjected to stress. Cell 125:1111-1124.

Boyerinas B, Park SM, Shomron N, Hedegaard MM, Vinther J, Andersen JS, Feig C, Xu J, Burge CB, Peter ME (2008) Identification of let-7regulated oncofetal genes. Cancer Res 68:2587-2591.

Brodsky M, Elliott K, Hynansky A, Inturrisi CE (1995) CNS levels of mu opioid receptor (MOR-1) mRNA during chronic treatment with morphine or naltrexone. Brain Res Bull 38:135-141.

Davis ME, Akera T, Brody TM (1979) Reduction of opiate binding to brainstem slices associated with the development of tolerance to morphine in rats. J Pharmacol Exp Ther 211:112-119.

Díaz A, Pazos A, Flórez J, Hurlé MA (2000) Autoradiographic mapping of mu-opioid receptors during opiate tolerance and supersensitivity in the rat central nervous system. Naunyn Schmiedebergs Arch Pharmacol 362:101-109.

Dum J, Meyer G, Höllt V, Herz A (1979) In vivo opiate binding unchanged in tolerant/dependent mice. Eur J Pharmacol 58:453-460.

Han W, Kasai S, Hata H, Takahashi T, Takamatsu Y, Yamamoto H, Uhl GR, Sora I, Ikeda K (2006) Intracisternal A-particle element in the 3 ' noncoding region of the mu-opioid receptor gene in CXBK mice: a new genetic mechanism underlying differences in opioid sensitivity. Pharmacogenet Genomics 16:451-460.

Holaday JW, Hitzemann RJ, Curell J, Tortella FC, Belenky GL (1982) Repeated electroconvulsive shock or chronic morphine treatment increases the number of ${ }^{3} \mathrm{H}$-D-Ala2,D-Leu5-enkephalin binding sites in rat brain membranes. Life Sci 31:2359-2362.

Höllt V, Dum J, Bläsig J, Schubert P, Herz A (1975) Comparison of in vivo and in vitro parameters of opiate receptor binding in naive and tolerant dependent rodents. Life Sci 16:1823-1828.

Ide S, Han W, Kasai S, Hata H, Sora I, Ikeda K (2005) Characterization of the $3^{\prime}$ untranslated region of the human mu-opioid receptor (MOR-1) mRNA. Gene 364:139-145.

Johnson EE, Chieng B, Napier I, Connor M (2006) Decreased mu-opioid receptor signalling and a reduction in calcium current density in sensory neurons from chronically morphine-treated mice. $\mathrm{Br} \mathrm{J}$ Pharmacol 148:947-955.

Kieffer BL, Evans CJ (2002) Opioid tolerance-in search of the holy grail. Cell 108:587-590

Klee WA, Streaty RA (1974) Narcotic receptor sites in morphine-dependent rats. Nature 248:61-63.

Koch T, Höllt V (2008) Role of receptor internalization in opioid tolerance and dependence. Pharmacol Ther 117:199-206.

Kosik KS (2006) The neuronal microRNA system. Nat Rev Neurosci 7:911-920.

Lewis JW, Lewis ME, Loomus DJ, Akil H (1984) Acute systemic administration of morphine selectively increases mu opioid receptor binding in the rat brain. Neuropeptides 5:117-120.

Mansfield JH, Harfe BD, Nissen R, Obenauer J, Srineel J, Chaudhuri A, Farzan-Kashani R, Zuker M, Pasquinelli AE, Ruvkun G, Sharp PA, Tabin CJ, McManus MT (2004) MicroRNA-responsive 'sensor' transgenes uncover Hox-like and other developmentally regulated patterns of vertebrate microRNA expression. Nat Genet 36:1079-1083.

Matthes HW, Maldonado R, Simonin F, Valverde O, Slowe S, Kitchen I, Befort K, Dierich A, Le Meur M, Dollé P, Tzavara E, Hanoune J, Roques
BP, Kieffer BL (1996) Loss of morphine-induced analgesia, reward effect and withdrawal symptoms in mice lacking the mu-opioid-receptor gene. Nature 383:819-823

Moss EG, Lee RC, Ambros V (1997) The cold shock domain protein LIN-28 controls developmental timing in C. elegans and is regulated by the lin- 4 RNA. Cell 88:637-646.

Mukhopadhyay D, Houchen CW, Kennedy S, Dieckgraefe BK, Anant S (2003) Coupled mRNA stabilization and translational silencing of cyclooxygenase- 2 by a novel RNA binding protein, CUGBP2. Mol Cell 11:113-126.

Obernosterer G, Martinez J, Alenius M (2007) Locked nucleic acid-based in situ detection of microRNAs in mouse tissue sections. Nat Protoc 2:1508-1514

Pillai RS, Bhattacharyya SN, Artus CG, Zoller T, Cougot N, Basyuk E, Bertrand E, Filipowicz W (2005) Inhibition of translational initiation by Let-7 MicroRNA in human cells. Science 309:1573-1576.

Reinhart BJ, Slack FJ, Basson M, Pasquinelli AE, Bettinger JC, Rougvie AE, Horvitz HR, Ruvkun G (2000) The 21-nucleotide let-7 RNA regulates developmental timing in Caenorhabditis elegans. Nature 403:901-906.

Rothman RB, Danks JA, Jacobson AE, Burke TR Jr, Rice KC, Tortella FC, Holaday JW (1986) Morphine tolerance increases mu-noncompetitive delta binding sites. Eur J Pharmacol 124:113-119.

Roush S, Slack FJ (2008) The let-7 family of microRNAs. Trends Cell Biol 18:505-516.

Sim LJ, Selley DE, Dworkin SI, Childers SR (1996) Effects of chronic morphine administration on mu opioid receptor-stimulated $\left[{ }^{35} \mathrm{~S}\right] \mathrm{GTPgam}-$ maS autoradiography in rat brain. J Neurosci 16:2684-2692.

Tang L, Shukla PK, Wang LX, Wang ZJ (2006) Reversal of morphine antinociceptive tolerance and dependence by the acute supraspinal inhibition of $\mathrm{Ca}(2+) /$ calmodulin-dependent protein kinase II. J Pharmacol Exp Ther 317:901-909.

Tao PL, Law PY, Loh HH (1987) Decrease in delta and mu opioid receptor binding capacity in rat brain after chronic etorphine treatment. J Pharmacol Exp Ther 240:809-816.

Tempel A, Habas J, Paredes W, Barr GA (1988) Morphine-induced downregulation of mu-opioid receptors in neonatal rat brain. Brain Res 469:129-133.

Wahlestedt C, Salmi P, Good L, Kela J, Johnsson T, Hökfelt T, Broberger C, Porreca F, Lai J, Ren K, Ossipov M, Koshkin A, Jakobsen N, Skouv J, Oerum H, Jacobsen MH, Wengel J (2000) Potent and nontoxic antisense oligonucleotides containing locked nucleic acids. Proc Natl Acad Sci U S A 97:5633-5638.

Wang JB, Johnson PS, Persico AM, Hawkins AL, Griffin CA, Uhl GR (1994) Human mu opiate receptor. cDNA and genomic clones, pharmacologic characterization and chromosomal assignment. FEBS Lett 338:217-222.

Wang ZJ, Wang LX (2006) Phosphorylation: a molecular switch in opioid tolerance. Life Sci 79:1681-1691.

Wong CS, Cherng CH, Luk HN, Ho ST, Tung CS (1996) Effects of NMDA receptor antagonists on inhibition of morphine tolerance in rats: binding at mu-opioid receptors. Eur J Pharmacol 297:27-33.

Wu L, Fan J, Belasco JG (2006) MicroRNAs direct rapid deadenylation of mRNA. Proc Natl Acad Sci U S A 103:4034-4039.

Wu Q, Law PY, Wei LN, Loh HH (2008) Post-transcriptional regulation of mouse mu opioid receptor (MOR1) via its $3^{\prime}$ untranslated region: a role for microRNA23b. FASEB J 22:4085-4095.

Wu Q, Zhang L, Law PY, Wei LN, Loh HH (2009) Long-term morphine treatment decreases the association of mu-opioid receptor (MOR1) mRNA with polysomes through miRNA23b. Mol Pharmacol 75:744750.

Yu VC, Sadée W (1988) Efficacy and tolerance of narcotic analgesics at the mu opioid receptor in differentiated human neuroblastoma cells. J Pharmacol Exp Ther 245:350-355.

Zadina JE, Chang SL, Ge LJ, Kastin AJ (1993) Mu opiate receptor downregulation by morphine and up-regulation by naloxone in SH-SY5Y human neuroblastoma cells. J Pharmacol Exp Ther 265:254-262.

Zöllner C, Johnson PS, Wang JB, Roy AJ Jr, Layton KM, Min Wu J, Surratt CK (2000) Control of mu opioid receptor expression by modification of cDNA 5' - and 3'-noncoding regions. Brain Res Mol Brain Res 79:159_ 162. 